\title{
OUTCOMES AND TREATMENT OF MALIGNANT TUMORS OF LONG BONES
}

doi: $10.2478 /$ rojost-2018-0064

\author{
G. Stan ${ }^{1,2}$, H. Orban ${ }^{1,2}$, N. Gheorghiu ${ }^{1,2}$, M. Drăguşanu ${ }^{1}$ \\ ${ }^{1}$ Elias University Hospital, Bucharest, Romania \\ 2"Carol Davila" University of Medicine and Pharmacy, Bucharest, Romania
}

Introduction. With the advances in chemotherapy, the life expectancy of patients with malignant tumors of bones begins to rise and creates the opportunity for a more conservative treatment of such cases. The main complications after using reconstruction with bone graft are represented by mechanical failure, due to the lack of strong fixation, or biological failure of graft integration. Infection is another complication, patients being usually immunosuppressed. The aim of this study was to investigate a retrospective single center experience of surgically treated malignant bone tumors of long bones, respectively oncological, surgical, and functional outcome differences after biological reconstruction.

Materials and methods. Between 2007 and 2017, we conducted a retrospective study, with patients from Elias Orthopaedic Department.

70 patients with malignant tumors were treated. Only in 20 cases, resection-reconstruction was possible.

Results. Survival rate at 8 -year follow up was $35 \%$. Mechanical failure rate was $20 \%$, graft resorption rate was $25 \%$, and infection rate was $15 \%$.

Discussion. Primary malignant tumors of bones are very rare, less than $1 \%$ of cancer cases, and yet there is no strict rule for treating these patients in specialized centers. Because of the small sample of patients, the heterogeneity of reconstruction methods and multiple types of grafts, this study also had some limitations. The mechanical or biological complication rate is higher in resectionreconstruction cases. Non-unions, fractures and infections are the most common complications. Keywords: malignant tumors, bones, resection-reconstruction 